\title{
VELOCITY FIELD COMPUTATION IN VIBRATED GRANULAR MEDIA USING AN OPTICAL FLOW BASED MULTISCALE IMAGE ANALYSIS METHOD
}

\author{
Johan Debayle ${ }^{1}$, Ahmed Raihane ${ }^{1}$, Abdelkrim BelhaOuA ${ }^{1}$, Olivier Bonnefoy ${ }^{1}$, \\ GÉrard ThOMAS ${ }^{1}$, JeAn-Marc ChaiX ${ }^{2}$ And JEAN-Charles PinOLI ${ }^{1}$ \\ ${ }^{1}$ École Nationale Supérieure des Mines de Saint-Etienne. Laboratoire des Procédés en Milieux Granulaires \\ (LPMG), UMR CNRS 5148. 158, cours Fauriel, 42023 Saint-Étienne cedex 2, France, ${ }^{2}$ SIMAP-LTPCM, \\ INPGrenoble-CNRS-UJF. BP 75 - Domaine Universitaire. 38402 Saint-Martin d'Hères, France \\ e-mail: debayle@emse.fr \\ (Accepted February 9, 2009)
}

\begin{abstract}
An image analysis method has been developed in order to compute the velocity field of a granular medium (sand grains, mean diameter $600 \mu \mathrm{m}$ ) submitted to different kinds of mechanical stresses. The differential method based on optical flow conservation consists in describing a dense motion field with vectors associated to each pixel. A multiscale, coarse-to-fine, analytical approach through tailor sized windows yields the best compromise between accuracy and robustness of the results, while enabling an acceptable computation time. The corresponding algorithm is presented and its validation discussed through different tests. The results of the validation tests of the proposed approach show that the method is satisfactory when attributing specific values to parameters in association with the size of the image analysis window. An application in the case of vibrated sand has been studied. An instrumented laboratory device provides sinusoidal vibrations and enables external optical observations of sand motion in 3D transparent boxes. At $50 \mathrm{~Hz}$, by increasing the relative acceleration $\Gamma$, the onset and development of two convective rolls can be observed. An ultra fast camera records the grain avalanches, and several pairs of images are analysed by the proposed method. The vertical velocity profiles are deduced and allow to precisely quantify the dimensions of the fluidized region as a function of $\Gamma$.
\end{abstract}

Keywords: grain motion, granular media, horizontal vibrations, multiscale image analysis, optical flow, velocity field.

\section{INTRODUCTION}

The aim of this work is to compute the velocity field of a granular medium submitted to different kinds of mechanical stresses. In particular, it focuses on the velocity field in a vibrated granular medium of sand grains (mean diameter $600 \mu \mathrm{m}$ ) and allows assessing the dimensions of its fluidized region, which corresponds to specific physical properties required in process engineering for industrial applications.

For this purpose, an image analysis method has been developed. 2D image sequences of the granular medium are firstly acquired with an ultrarapid CCD camera. The optical flow (dense velocity field) between two images is then computed using a differential method. This technique is based on two assumptions. The first one is the light intensity conservation, implying that the luminance of the grains does not change significantly between two successive image frames at time values $t$ and $t+\mathrm{d} t$.
The second one requires that each velocity vector remains constant within a small spatial window. The size of the analyzing window is related to the accuracy of the method. In order to handle small and large displacements, the optical flow is computed with an iterative and multiscale approach (pyramidal decomposition) using a coarse-to-fine analysis. The level of the pyramid multiscale analysis is related to the robustness of the method. In order to get the best compromise between accuracy and robustness, several validation tests are achieved. For a given numerical or physical motion (translation, rotation), pairs of images before and after the displacement are compared. Using the optimal parameters, the image analysis method is performed in the framework of a study of an horizontally vibrated granular medium submitted to different accelerations. The vertical velocity profiles are then deduced and allow to precisely quantify the dimensions of the fluidized region as a function of the vibration acceleration. 


\section{MATERIALS, EXPERIMENTAL SETUPS AND IMAGE ACQUISITION}

\section{MATERIALS}

The granular medium is made of sand grains (more than $99 \%$ of $\alpha$-quartz) without internal close porosity $\left(2660 \mathrm{~kg} / \mathrm{m}^{3}\right)$. The size distribution given on Fig. 1 shows a moderate polydisperse material with a span of $\left(d_{90 \%}-d_{10 \%}\right) / d_{50 \%}=0.75$ and a volume mean diameter, denoted $d_{4,3}$ (the ratio of the fourth to the third moments of the grain size distribution), of $600 \mu \mathrm{m}$. The grains are approximately spherical (Fig. 2).

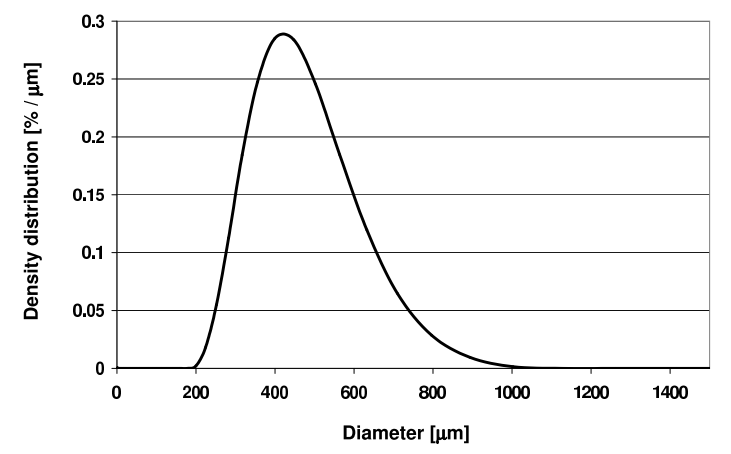

Fig. 1. Volumetric grain size distribution. The mean diameter is $d_{4,3}=600 \mu \mathrm{m}$ with a span of $\left(d_{90 \%}-\right.$ $\left.d_{10 \%}\right) / d_{50 \%}=0.75$ where $n \%$ in mass of the grains have diameter lower than $d_{n \%}$.

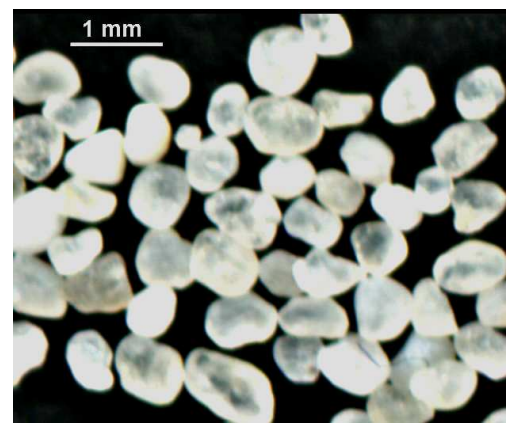

Fig. 2. Optical microscope image showing the rounded shapes of the sand grains.

\section{EXPERIMENTAL SETUPS}

Three experimental setups have been used.

Translation bench. The translation bench is composed of a parallelepipedic open box (container) filled with sand. This container is fixed on an horizontal linear motion guide, allowing translational motion exclusively. The camera is placed above the setup.
Rotation bench. This setup is composed of a plastic cap of $3.3 \mathrm{~cm}$ inner diameter with the openside up. A pushpin goes through it center and fixes it on an horizontal plank, allowing rotational motion exclusively. The container is filled with sand grains and the camera is placed above the setup, aligned with the pushpin's vertical axis (Fig. 3).

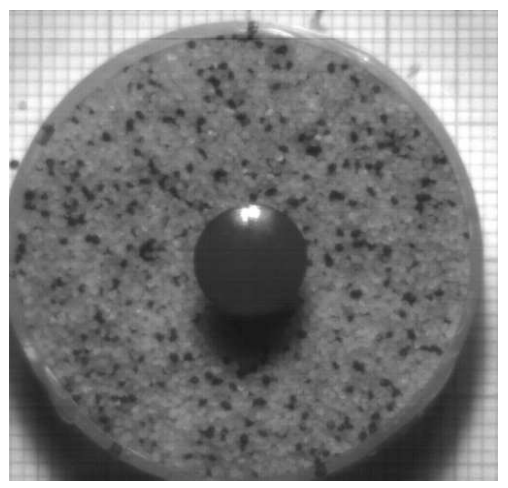

Fig. 3. Rotation bench: a plastic cap filled with granular material can undergo a rotational motion around its center where a pushpin is nailed.

Vibration bench. This experimental setup (Raihane et al., in press) is mainly composed of a mobile table, a transparent container and sand grains. The horizontal table is linked to a marble stand by four horizontal linear motion guides (THK, EPF7M16+55LM). A sinusoidal motion is transmitted to the table by an electromagnetic shaker (TIRA, S513) driven by a signal generator (LING DYNAMIC SYSTEMS, DSC4) coupled with a power amplifier (TIRA, BAA120). The motion is driven with an accelerometer (BRUEL \& KJAER, $4371 \mathrm{~V})$. The direction of the vibrations is referred to as $x$-axis. The parallelepipedic container fixed on the table is composed of $8 \mathrm{~mm}$ thick glued plexiglas plates (Fig. 4).

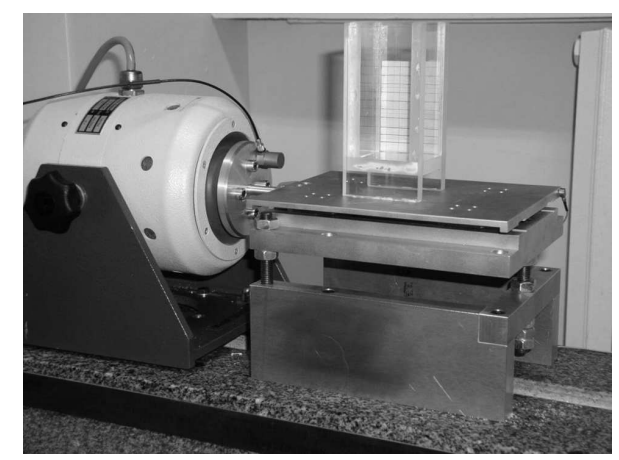

Fig. 4. Vibration bench: an electromagnetic shaker delivers a sinusoidal vibration at a controled frequency $f$. It drives an horizontal table mounted on four bearings guided by four rails. The vibration amplitude $A$ is controlled by a piezoelectric accelerometer. 
The longitudinal, transverse and vertical dimensions $L_{x}, L_{y}, L_{z}$ of the container are respectively 40,80 and $80 \mathrm{~mm}$. The initial height of the granular packing is $60 \mathrm{~mm}$ and the camera observes the vertical face orthogonal to the vibration $x$-axis.

\section{IMAGE ACQUISITION}

An ultra-fast CCD camera (JAI, CMOS CV-A33) with a maximal frequency of 5400 frames/s and a maximal field of $494 \times 660$ pixels allow us to follow the movements of the grains along the walls of the box. The analysis of the images sequences is described below.

\section{VELOCITY FIELD COMPUTATION}

This section is devoted to an image analysis method allowing the computation of a dense velocity field from an image sequence of vibrated granular structures. For that, an optical flow multiscale method is proposed.

\section{OPTICAL FLOW BASED IMAGE ANALYSIS METHOD}

Optical flow (Horn and Schunck, 1981) is an estimation of the apparent motion (velocity) of objects within an image sequence. It is closely related to motion estimation. Nevertheless, the term optical flow is specifically used to describe a dense $2 \mathrm{D}$ apparent motion field from the projection of a 3D scene onto the image plane. Different methods have been reported for determining optical flow such as:

- phase-based methods (Fleet and Jepson, 1990; Fleet, 1992): the velocity is defined in terms of the phase behavior of band-pass filter outputs. They provide a high accuracy but are generally less efficient for large displacements;

- differential methods (Horn and Schunck, 1981; Lucas and Kanade, 1981; Uras et al., 1988): velocity is computed from spatio-temporal derivatives of image intensities. These techniques offer a good trade-off between robustness under noise and density of the flow fields;

- region-based matching methods (Burt et al., 1983; Anandan, 1989; Little and Verri, 1989): the velocity is defined as the shift yielding the best fit between image regions, according to some similarity measure. These methods provide more robustness with respect to differentiation and are generally quicker but they are less successful for sub-pixel velocities;
- energy-based methods (Adelson and Bergen, 1986; Heeger, 1988; Barman et al., 1991): optical flow is computed using the output from the energy of velocity tuned filters in the Fourier domain. It has been shown that certain energy-based methods are equivalent to region-based or differentiation methods.

In review papers, Barron et al. (1994) and Galvin et al. (1998) have evaluated different methods and concluded that the differential method proposed by Lucas and Kanade (1981) yields the best results.

Other numerous methods combining local (using spatial constancy assumptions) and global (using smoothness assumptions) techniques have also been developed so as to propose an accurate smooth and dense optical flow field (Brox et al., 2004; Bruhn and Weickert, 2005; Le Besnerais and Champagnat, 2005). For the proposed application, a dense optical flow is not absolutely necessary and a raw velocity field is first required. Therefore, the computation of the velocity field in this paper is based on the differential method proposed by Lucas and Kanade (1981).

The Lucas and Kanade (1981) method, as many differential techniques, is based on the assumption of intensity conservation (i.e., assuming that the intensity of the objects within the image sequence $I$ does not change significantly between two successive image frames at times $t$ and $t+\mathrm{d} t$ ), that is to say:

$$
I(x, y, t)=I(x+\mathrm{d} x, y+\mathrm{d} y, t+\mathrm{d} t),
$$

where ${ }^{t}(x, y)$ and ${ }^{t}(\mathrm{~d} x, \mathrm{~d} t)$ are vectors of the pixel location and displacement respectively; ${ }^{t}$ denoting the matrix (vector) transposition.

For small displacements, a first order Taylor expansion can be applied:

$$
\begin{aligned}
& I(x+\mathrm{d} x, y+\mathrm{d} y, t+\mathrm{d} t)=I(x, y, t)+ \\
& \quad \frac{\partial I}{\partial x}(x, y, t) \mathrm{d} x+\frac{\partial I}{\partial y}(x, y, t) \mathrm{d} y+\frac{\partial I}{\partial t}(x, y, t) \mathrm{d} t .
\end{aligned}
$$

Combining Eq. 1 and Eq. 2, the optical flow constraint is then given as:

$$
\frac{\partial I}{\partial x}(x, y, t) \mathrm{d} x+\frac{\partial I}{\partial y}(x, y, t) \mathrm{d} y+\frac{\partial I}{\partial t}(x, y, t) \mathrm{d} t=0,
$$

which results in:

$$
\frac{\partial I}{\partial x}(x, y, t) v_{x}+\frac{\partial I}{\partial y}(x, y, t) v_{y}+\frac{\partial I}{\partial t}(x, y, t)=0,
$$

where $v={ }^{t}\left(v_{x}, v_{y}\right)$ is the velocity vector at location and time $(x, y, t)$. 
Thus, Eq. 4 could be written as the differential system:

$$
\left(I_{x}, I_{y}\right)^{t}\left(v_{x}, v_{y}\right)+I_{t}=0,
$$

where $I_{x}, I_{y}$ and $I_{t}$ denote the space and time partial derivative of $I(x, y, t)$, respectively.

This is a system of one equation with two unknowns $v_{x}, v_{y}$ ant this it can not be solved as such. This is known as the aperture problem of the optical flow algorithms. Consequently, another set of equations is needed, given by some additional constraints (Horn and Schunck, 1981).

To solve the aperture problem, Lucas and Kanade (1981) assume a locally constant velocity in the neighborhood of the considered pixel. Assuming that the velocity vector $v={ }^{t}\left(v_{x}, v_{y}\right)$ is constant within a small window $W$ of size $n$ pixels centered at $(x, y)$ and ordering the pixels within as $1, \ldots, n$, yields an overdetermined system:

$$
\left[\begin{array}{cc}
I_{x_{1}} & I_{y_{1}} \\
I_{x_{2}} & I_{y_{1}} \\
\vdots & \vdots \\
I_{x_{n}} & I_{y_{n}}
\end{array}\right]\left[\begin{array}{l}
v_{x} \\
v_{y}
\end{array}\right]+\left[\begin{array}{c}
I_{t_{1}} \\
I_{t_{2}} \\
\vdots \\
I_{t_{n}}
\end{array}\right]=0
$$

This system can be written in the following matricial form:

$$
A v+b=0,
$$

where $A, v, b$ denote the space partial derivative matrix, the velocity vector and the time partial derivative vector, respectively.

To solve this system of equations, the least squares method is used in the Lucas-Kanade optical flow estimation:

$$
v=\left({ }^{t} A A\right)^{-1}\left[{ }^{t} A \cdot(-b)\right],
$$

that is to say:

$$
\left[\begin{array}{l}
v_{x} \\
v_{y}
\end{array}\right]=\left[\begin{array}{cc}
\sum_{i=1}^{n} I_{x_{i}}^{2} & \sum_{i=1}^{n} I_{x_{i}} I_{y_{i}} \\
\sum_{i=1}^{n} I_{x_{i}} I_{y_{i}} & \sum_{i=1}^{n} I_{y_{i}}^{2}
\end{array}\right]^{-1}\left[\begin{array}{c}
-\sum_{i=1}^{n} I_{x_{i}} I_{t_{i}} \\
-\sum_{i=1}^{n} I_{y_{i}} I_{t_{i}}
\end{array}\right] .
$$

This estimation is reliable if the matrix ${ }^{t} A A$ is invertible, i.e., with no zero eigenvalues. Consequently, the Lucas and Kanade (1981) method computes the vector $v$ at a pixel $(x, y)$ only if both eigenvalues $\lambda_{1}, \lambda_{2}$ of ${ }^{t} A A$ are greater than a predefined threshold value $\lambda$. Note that if $\lambda_{1}, \lambda_{2}$ are both large, the point $(x, y)$ corresponds to a corner point (Harris and Stephens, 1988). Therefore, in the proposed method, the velocity vectors are computed at corner points.

\section{MULTISCALE APPROACH}

The two key components of an optical flow method are accuracy and robustness. These components relate to the size of the window $W$ :

- a small window is preferable for accuracy in order not to "smooth out" the details contained in the images,

- a large window is preferable for robustness to handle large motions.

There is therefore a compromise between accuracy and robustness when choosing the window size. In this way, a multiscale and iterative implementation (Bouguet, 2000) is proposed for providing both a robust and accurate method.

The multiscale representation is given by a Gaussian pyramid (Burt and Adelson, 1983). In this way, the optical flow is computed in a coarse-tofine analysis. The velocity is first computed at a low resolution (i.e., at a high level of the pyramid), in an iterative way so as to ensure the stabilization of the solution, before advancing to the next level. Then, the field is warped and upsampled at the next level of the pyramid and the optical flow is computed again. This loop estimation is performed until the highest image resolution (i.e., at the lowest level of the pyramid).

This iterative multiscale algorithm, denoted Motion-2D (Belhaoua, 2007), for estimating the optical flow between two image frames is therefore decomposed in the steps presented in Alg. 1.

Note that all computations are achieved at a subpixel accuracy level, using bilinear interpolation.

\section{REGULARIZATION}

Despite that velocity vectors are only computed for large eigenvalues of ${ }^{t} A A$ (see section about optical flow), some outliers could appear. So, in order to have a smoothed optical flow (i.e., to discard outliers), a median filtering could be applied on the dense motion field (using either the norm or the angle of the vectors).

In the proposed algorithm, the median filtering is only performed if the norm or the angle of the considered vector $v={ }^{t}\left(v_{x}, v_{y}\right)$ is far from the median value of those within a $3 \times 3$ window centered on the pixel located at $(x, y)$.

This regularization process has been added to Motion-2D as an option. 


\footnotetext{
input : Images $I$ and $J$

output: Velocity field between images $I$ and $J$

pyramidal decomposition in $L+1$ levels of the two image frames $I$ and $J: I_{0}=I, I_{1}, \ldots, I_{L}$ and $J_{0}=J, J_{1}, \ldots, J_{L}$;

initialization of the optical flow: $v_{L+1}=0$;

for $l \leftarrow L$ to 0 do

image $I_{l}^{+}$: warping of the image $I_{l}$ with the upsampled optical flow $v_{l+1}^{*}$ :

$I_{l}^{+}=I_{l}+v_{l+1}^{*}$

loop estimation of the optical flow between images $I_{l}^{+}$and $J$ :

for $k \leftarrow 1$ to $K$ do $\eta_{l}^{k} \equiv$ iterative Lucas-Kanade. $\eta_{l}^{k}$ is the optical flow at level $l$ for iteration $k$. end

optical flow at level $l$ between images $I_{l}^{+}$ and $J: \eta_{l}$

final optical flow at level $l$ between images $I_{l}$ and $J: v_{l}=v_{l+1}^{*}+\eta_{l}$;

end

estimation of the final optical flow between images $I$ and $J$ :

$v=v_{0}=\sum_{l=0}^{L} \eta_{l}^{*}$

$\eta_{l}^{*}$ is the optical flow $\eta_{l+1}$ upsampled at level $l$;
}

Algorithm 1: Algorithm Motion-2D. The velocity field is computed using an iterative multiscale image analysis method.

\section{VALIDATION}

In general, the performance of the optical flow methods are evaluated on real sequences and synthetic sequences for which motion fields are known. Some common evaluation criteria are average angular error, standard deviation and density of measurements (Barron et al., 1994).

\section{GENERATION OF TEST IMAGES}

In order to validate the implementation of the proposed algorithm in terms of accuracy and robustness, several tests have been performed on images of granular structures with imposed motion. Four pairs of images have been built:

- $A-B$ (numerical test / translation): A picture $A$ of a sand packing is shifted by exactly $p$ pixels with Aphelion $^{\mathrm{TM}}$ software to give a picture $B$.
- $C-D$ (numerical test / differential translation): A picture $C$ of a sand packing is split into different zones $C_{i}$ which are shifted by $p_{i}$ pixels each to give a picture $D$.

- $E-F$ (physical test / translation): The sand packing is shifted by $d \mathrm{~mm}$ with the translation bench. The pictures of the initial and final states are labeled $E$ and $F$ respectively.

- $G-H$ (physical test / rotation): The sand packing is rotated by an angle of $\alpha$ degrees with the rotation bench. The pictures of the initial and final states are labeled $G$ and $H$ respectively.

\section{VELOCITY FIELD COMPUTATION FOR TEST IMAGES}

$A-B$ : Various pairs of images $A-B$ were created corresponding to translations between one and ten pixels. Several analyses were performed on these images by varying the level of pyramid $L$ from 0 to 4 and the window size $W$ from 3 to 25 pixels. Results of these analyzes are presented on Fig. 5. They show that there exists conditions for a displacement of $p$ pixels to be accurately estimated. In fact, when using small window $(W<p)$, analyzes should be carried out for high pyramid levels. For instance, for a displacement of ten pixels, one shall use $L=3$ (pyramid level) and $W=3$ (window size). Let us notice that in these experiments, a mean grain diameter is equal to about eight pixels. So, the width of the largest window $W$ is about three mean diameters. Computation time is an other important parameter especially for quantitative analyses. This parameter is almost constant when increasing pyramid level for small windows $(W<9)$ and increases dramatically for large windows $(W>9)$. Results obtained for pyramid level $L=2$ are satisfactory for all the investigated window sizes. For the following analyzes, the pyramid level $L$ is kept equal to two and the window width $W$ is chosen to be equal to three grain's mean diameter $d_{4,3}$. The latter choice will be justified in the section Results. 


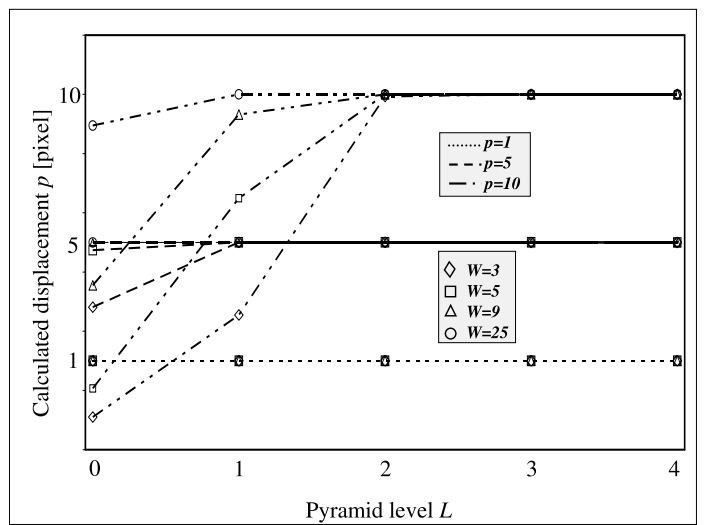

Fig. 5. Numerical test: the computed displacement (in pixels) between two images $A$ and $B$ (for different numerical translations) is plotted versus the pyramid level $L$ for different window sizes $W$.

$C-D$ : Using a pyramid level $L=2$ and a window width $W \approx 3 d_{4,3}$, the displacement field between images $C$ and $D$ was calculated. For each z, the displacement is averaged along the $x$-axis. The resulting profile is plotted on Fig. 6. The different regions corresponding to different values of displacement $p$ pixels are well detected and separated.

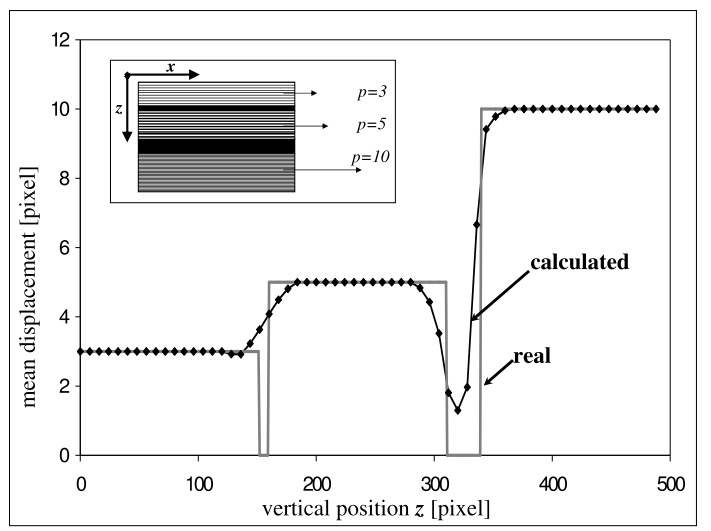

Fig. 6. Differential numerical test: the mean computed and real displacements between two images $C$ and $D$ are plotted versus the vertical position z. The thick (resp. thin) line represents the computed (resp. real) displacement. The inset shows the different translations performed on image $C$ to obtain image $D$.

$E-F$ : Several translation tests, from $0.1 \mathrm{~mm}$ to $1.5 \mathrm{~mm}$, were performed. The resulting images were analyzed with parameters $L=2$ and $W \approx$ $3 d_{4,3}$. Table 1 shows the experimental results and calculated results.
Table 1. Physical translation: Comparison between real and $p_{\text {calculated }}$ displacement of a sand packing. The image analysis method is performed with parameters $L=2$ and $W=15$.

\begin{tabular}{lcc}
\hline$d[\mathrm{~mm}]$ & $d$ [pixel] & $p_{\text {calculated }}$ \\
\hline 0.1 & 0.82 & $0.83 \pm 0.02$ \\
0.3 & 2.46 & $2.49 \pm 0.02$ \\
0.4 & 3.28 & $3.41 \pm 0.03$ \\
0.5 & 4.1 & $4.2 \pm 0.09$ \\
0.8 & 6.56 & $6.63 \pm 0.04$ \\
1. & 8.2 & $8.29 \pm 0.09$ \\
1.5 & 12.3 & $12.08 \pm 0.3$ \\
\hline
\end{tabular}

$G-H$ : Fig. 7 shows the result of the rotation test analysis by Motion-2D. By going away from the center of rotation (pushpin center), the displacement increases according to the law $d=$ $\alpha \times r$ (r: radius).

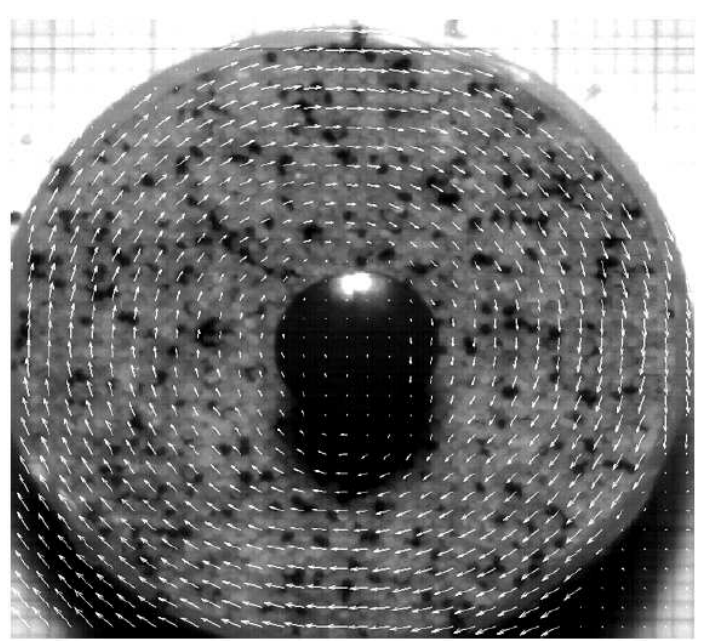

Fig. 7. Displacement field from rotation: the cup filled with granular material was rotated around the pushpin. The resulting images were analyzed with parameters $L=2$ and $W=15 \equiv 3 D_{4,3}$.

Following these validation experiments, the algorithm is parameterized with a pyramidal level $L=2$ levels, a number of 100 iterations and a window size $W$ of approximately three mean grain diameters.

\section{RESULTS}

\section{EXPERIMENTAL OBSERVATIONS}

In vibration experiments, a typical run consists in increasing the value of the relative acceleration $\Gamma$ from zero at a fixed frequency $f=50 \mathrm{~Hz}$. Different behaviors were observed as a function of $\Gamma$. To facilitate the discussion, the vertical faces orthogonal 
(resp. parallel) to the vibrations direction are labeled "North/South" (resp. "East/West"). The observations are the following ones (Raihane et al., in press):

$0<\Gamma<\Gamma_{1} \sim 0.3$ : The whole granular packing behaves like a compact solid which follows the motion of the box (at rest in the box frame).

$\Gamma_{1}<\Gamma<\Gamma_{2} \sim 0.9$ : Grains simmering at the free surface; the other regions are at rest in the box frame.

$\Gamma_{2}<\Gamma<4.5$ : At $\Gamma_{2}$, a transition occurs from a solidlike behavior of the whole packing to a fluidlike one in the upper region, the lower region remaining unchanged. At the free surface, grains move towards the walls from the middle of the granular medium and then, avalanche into the gap created between the granular packing and the North/South walls. On the East/West faces, two convective counter-rotating rolls are observed (Fig. 8). Only the upper region of the packing is fluidized. By increasing the relative acceleration $\Gamma$, the thickness of the fluidized region $E_{f}$ increases.

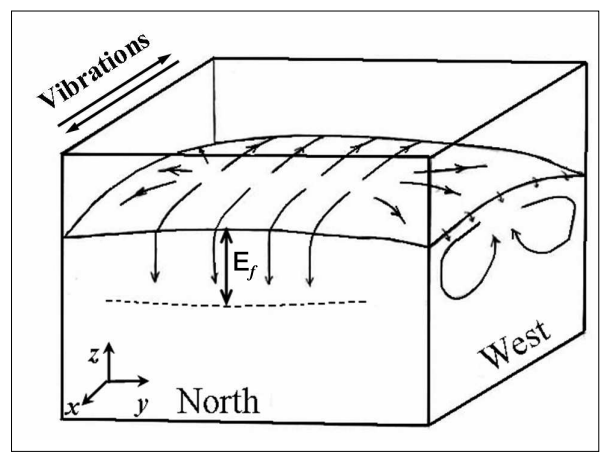

Fig. 8. Convective rolls: sketch of convective motion in a granular packing submitted to horizontal and sinusoidal vibrations with acceleration $\Gamma>1$. In the west/east faces, two counter rotating convective rolls are observed. At the north/south walls, grains avalanche to a distance $E_{f}$ from the top.

\section{QUANTITATIVE DESCRIPTION}

In vibration experiments, the aim is to measure the velocity field of grains at the North/South faces and the fluidized region thickness $E_{f}$. For this purpose, a $\mathrm{CCD}$ camera records the grain avalanches at the north face at the rate of one frame per oscillation period (50 frames per second). For each acceleration, several pairs of images are analyzed by Motion-2D (10 pairs for $\Gamma \leq$ 1.8, 30 pairs else). The resulting displacement fields are averaged and then, for each acceleration, a vertical velocity profile is deduced (Fig. 9). For all these analyses, the pyramid level is $L=2$ and the window width $W \approx 3 d_{4,3}$. The choice of such window width is justified by the fact that, during their fall, grains can undergo simultaneously rotation motions. Hence, the algorithm would meet difficulties to recognize them if the window recovers only one grain. A window width of three grain mean diameters seems to be a good compromise to avoid chaotic behavior of granular movements and to take into account the displacement gradient (Medved, 2002).

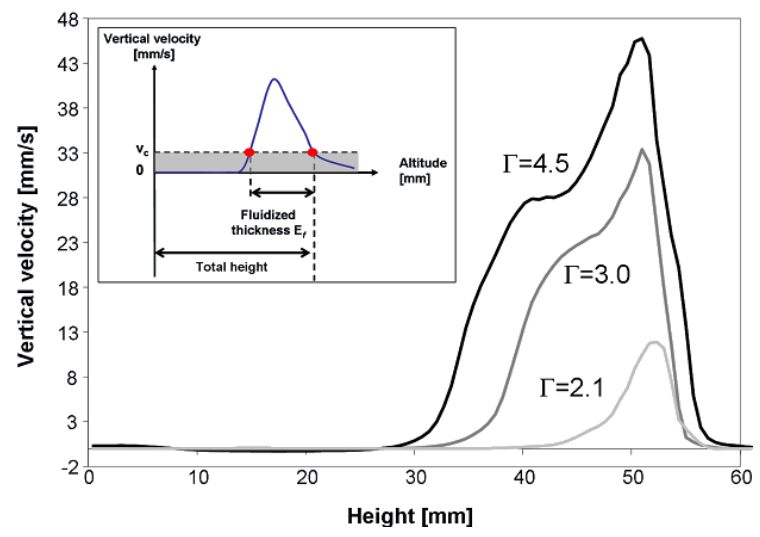

Fig. 9. Velocity profiles of grains at the North wall for a granular packing of initial height $H_{i}=60 \mathrm{~mm}$ horizontally vibrated at three different accelerations. Profiles show a flat zone and a large peak corresponding respectively to the solid lower zone and the fluidized upper zone of the packing.

To determine the thickness of the fluidized region for each acceleration, a fluidization velocity threshold is defined (inset of Fig. 9). Here, when the threshold of $0.06 \mathrm{~mm} / \mathrm{s}$ is exceeded, the granular medium is said to be fluidized.

Fig. 10 shows the evolution of the fluidized thickness $E_{f}$ versus the relative acceleration $\Gamma$ for a granular packing vibrated at frequency $f=50 \mathrm{~Hz}$.

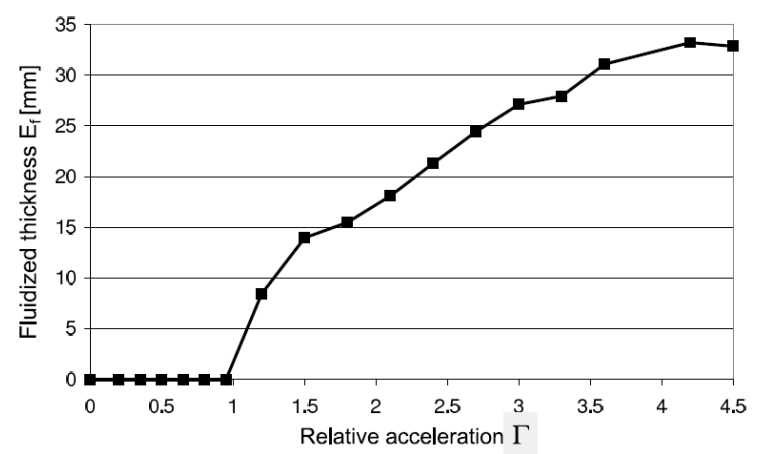

Fig. 10. Evolution of the fluidized thickness with relative acceleration $\Gamma$ of a granular packing of initial height $H_{i}=60 \mathrm{~mm}$. An onset of fluidization is observed for $\Gamma=1$ and a saturation for high accelerations. 
The phenomena occuring on East and West faces can be described in the same way with Motion-2D.

Fig. 11 shows the computed displacement field for an image sequence during $20 \mathrm{~s}$.

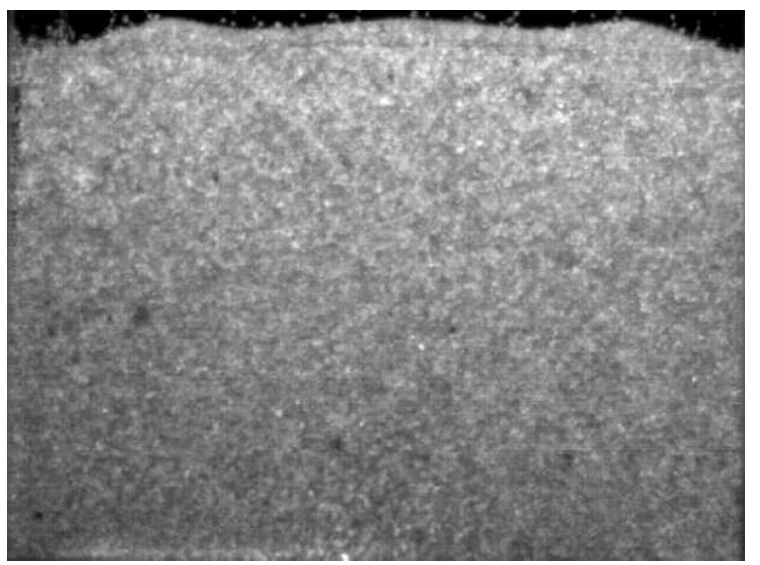

(a) image at $t=20 \mathrm{~s}$

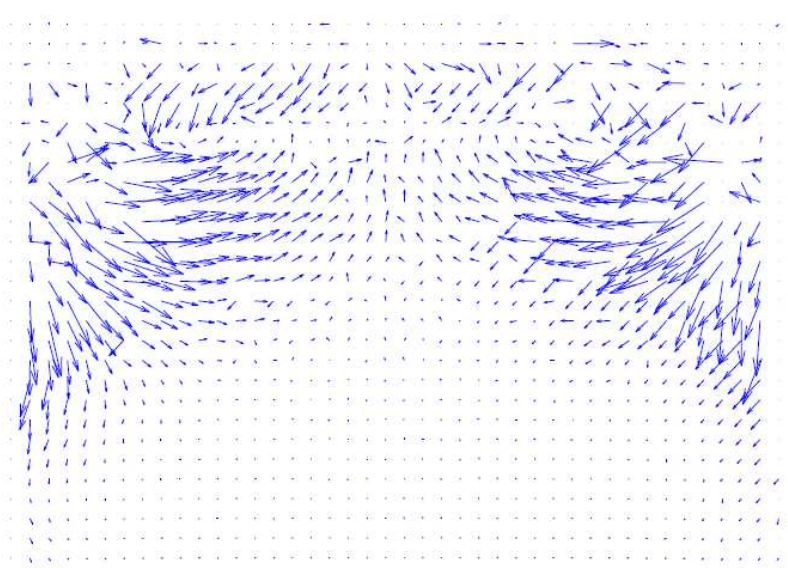

(b) velocity vector field

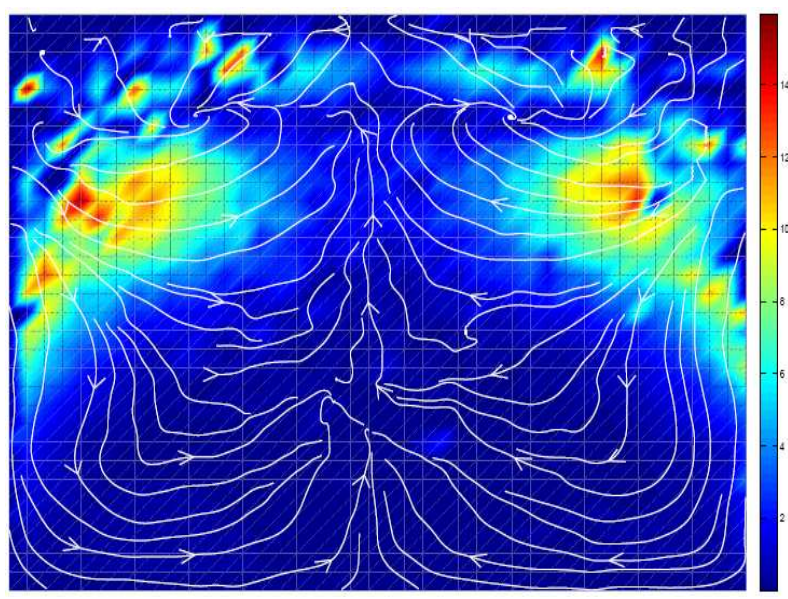

(c) velocity streamlines

Fig. 11. Computed velocity field of a granular medium vibrated during $20 \mathrm{~s}$. The vector velocity field $(b)$ is computed between images from $t=0 \mathrm{~s}$ to $t=20 \mathrm{~s}$ (a). The streamlines (c) shows the direction and the magnitude of the flow velocity.
The velocity field shows two convective rolls in the left and right upper corners of the image. This numerical result is in accordance with the experimental observations (Fig. 8).

Finally, this quantitative description using Motion$2 D$ provides the velocity field in a vibrated sand granular packing and therefore enables to estimate its fluidized thickness.

\section{CONCLUSION}

An optical flow based multiscale image analysis method has been developed in order to compute the velocity field in vibrated granular media constituted of sand grains. This method provides a good compromise between accuracy and robustness for acceptable computation time, using specific parameters assessed from several validation tests. The velocity vectors are computed both using tailor sized windows and a coarse-to-fine analysis on 2D image sequences. The results provide a quantification of the fluidized thickness of the granular medium versus the relative acceleration of the vibration (using a fixed frequency).

\section{REFERENCES}

Adelson EH, Bergen JR (1986). The extraction of spatiotemporal energy in human and machine vision. In: Proc IEEE Worksh Visual Motion. Charleston, SC, USA; May, 151-66.

Anandan P (1989). A computational framework and an algorithm for the measurement of visual motion. Int J Comput Vision 2:283-310.

Barman H, Haglund L, Knutsson H, Granlund G (1991). Estimation of velocity, acceleration and disparity in time sequences. In: Proc IEEE Motion Worksh. Princeton, NJ, USA; October, 44-51.

Barron JL, Fleet DJ, Beauchemin SS, Burkitt TA (1994). Performance of optical flow techniques. Int J Comput Vision 12:43-77.

Belhaoua A (2007). Analyse et modélisation dynamique d'images de structures granulaires soumises à des vibrations. MSc Thesis, ENSM-SE, Saint-Etienne, France.

Bouguet JY (2000). Pyramidal implementation of the lucaskanade feature tracker. Tech. rep., Intel Corporation, Microprocessor Research Labs.

Brox T, Bruhn A, Papenberg N, Weickert J (2004). High Accuracy Optical Flow Estimation Based on a theory for warping. In: Proc 8th Eur Conf Comput Vision. Prague, Czech Republic; May, 25-36, vol. 4. 
Bruhn A, Weickert J (2005). Lucas/Kanade Meets Horn/Schunck: Combining Local and Global Optic Flow Methods. Int J Comput Vision 61:211-31.

Burt P, Yen C, Xu X (1983). Multiresolution flow-through motion analysis. In: Proc IEEE Int Conf Comput Vision Pattern Recogn. Washington, DC, USA; June, 246-52.

Burt PJ, Adelson EH (1983). The laplacian pyramid as a compact image code. IEEE Trans Commun 31:532-40.

Fleet D, Jepson A (1990). Computation of component image velocity from local phase information. Int $\mathbf{J}$ Comput Vision 5:77-104.

Fleet DJ (1992). Measurement of Image Velocity. Kluwer Academic Publishers, Norwell.

Galvin B, McCane B, Novins K, Mills S (1998). Recovering motion fields: An evaluation of eight optical flow algorithms. In: Proc Brit Machine Vision Conf. Southampton, UK; September, 195-204.

Harris C, Stephens MJ (1988). A combined corner and edge detector. In: Proc Alvey Vision Conf. Manchester, UK; September, 147-52.
Heeger DJ (1988). Optical flow using spatiotemporal filters. Int J Comput Vision 1:279-306.

Horn BKP, Schunck BG (1981). Determinig optical flow. Artif Intel 17:185-203.

Le Besnerais G, Champagnat F (2005). Dense optical flow by iterative local window registration. In: Proc IEEE Int Conf Image Proc. Genova, Italy. September, 137-40.

Little JJ, Verri A (1989). Analysis of differential and matching methods for optical flow. In: IEEE Worksh Visual Motion. Irvine, CA, USA; March, 173-180.

Lucas BD, Kanade T (1981). An iterative image registration technique with an application to stereo vision. In: Proc Int Joint Conf Artif Intel. Vancouver, BC, Canada; August, 674-9.

Medved M (2002). Connections between response modes in a horizontally driven granular material. Phys Rev E 65:021305.

Uras S, Girosi F, Verri A, Torre V (1988). A computational approach to motion perception. Biol Cybern 60:79-97. 